\title{
Evaluation of Left Ventricular Diastolic Function According to New Criteria and Determinants in Acromegaly
}

\author{
Bahri Akdeniz, ${ }^{1}$ MD, Arzu GediK, ${ }^{2}$ MD, Onur Turan, ${ }^{3} \mathrm{MD}$, Ebru OzPelit, ${ }^{1} \mathrm{MD}$, Ahmet Omer Ikiz, ${ }^{4} \mathrm{MD}$, \\ Oya ITIL, ${ }^{3} \mathrm{MD}$, Ozer BADAK, ${ }^{1} \mathrm{MD}$, Nezihi BARIs, ${ }^{1} \mathrm{MD}$, and Abdurrahman ÇöMLEKÇI, ${ }^{2} \mathrm{MD}$
}

\begin{abstract}
SUMMARY
Left ventricular diastolic dysfunction (LVDD) develops in the early stages of acromegaly. The purpose of this study was to identify LVDD analyzing by new echocardiograpic criteria as well as to evaluate determinants of the LVDD in acromegaly. This cross-sectional study examined 42 patients with acromegaly; 16 in active disease (AA) and 26 cured/ well controlled (CA), and compared them with 30 healthy controls (CG). Ventricular systolic and diastolic functions were studied by conventional and tissue Doppler imaging based on the E/Em ratio and myocardial performance index (MPI). Other clinical parameters possibly contributing to LVDD in acromegaly were also investigated. The prevalence of LV hypertrophy (33\%) and LVDD (35.7\%) were increased in acromegaly, however, there were no differences between the AA and CA groups. Acromegalic patients had higher LV volumes and LV mass, and septal E/Em ratio compared to CG, whereas LV ejection fraction and MPI were not different. The presence of acromegaly $(r=0.29, P=0.013)$, diabetes mellitus $(\mathrm{DM})(r=0.41, P<0.001)$, hypertension $(r=0.35, P=0.002)$, and sleep apnea $(r=0.56, P=0.003)$ were found to be correlated with LVDD, whereas duration and activity of acromegaly were not. In regression analysis, advanced age (OR: 8.53, $P=0.006)$ and DM (OR: 25.9, $P=0.007)$ were found to be independent risk factors for LVDD. The risk of LVDD according to new criteria increases in acromegaly. However, it seems to be related to the presence of DM and advanced age and is independent of disease duration and activity. (Int Heart J 2012; 53: 299-305)
\end{abstract}

Key words: Left ventricular function, Diabetes mellitus, Left ventricular hypertrophy, Echocardiography, Doppler

A cromegaly is characterized by an excess of growth hormone $(\mathrm{GH})$ secretion, which stimulates the growth of various tissues including the cardiovascular system. ${ }^{1)}$ Cardiac involvement is known to be a major determinant of the shortened life expectancy in acromegalic patients. ${ }^{1,2)}$ Increased $\mathrm{GH}$, directly and via insulin like growth factor 1 (IGF-1), induces myocardial hypertrophy and fibrosis in the absence of increased wall stress. ${ }^{3)}$ Left ventricular hypertrophy (LVH) causes diastolic and more rarely systolic dysfunction and arrhythmias. Echocardiographic studies of acromegalic patients have suggested left ventricular diastolic dysfunction (LVDD) in the early stages of the disease while LV systolic function was preserved. ${ }^{4}$ However, previous studies have evaluated diastolic function by old methods including the transmitral E/A ratio. This ratio is not sufficient to evaluate the diastolic function in patients with preserved systolic function. Pulsed-wave (pw) tissue Doppler imaging (TDI) allows quantitative measurements of the myocardial contraction and relaxation velocities of a selected myocardial segment. ${ }^{5)}$ The Em velocity is considered as an index of ventricular relaxation, which is relatively independent from variation of the atrial pressure and its ratio to velocity peak of transmitral flow $(\mathrm{E} / \mathrm{Em})$ is related with the LV filling pressure. The E/Em ratio is recommended at the first step for evaluation of LVDD by echocardiography according to a new guideline. ${ }^{6}$ Peak systolic mitral annular velocity
(Sm) is used to evaluate LV systolic function. Another parameter used in the evaluation of cardiac function is the myocardial performance index (MPI). MPI may give a better reflection of the global LV function rather than an isolated evaluation of either ejection or relaxation. ${ }^{7)}$ It is obtained from the sum of the isovolumetric relaxation and contraction time divided by ejection time. When its value is over 0.45 , it indicates cardiac dysfunction and suggests future heart failure.

On the other hand, hypertension, diabetes mellitus (DM), and sleep disorders (obstructive sleep apnea syndrome; OSAS) frequently coexist with acromegaly. ${ }^{2,89}$ The cardiac involvement in acromegaly may be due to the combined effects of both these comorbid disorders and acromegaly itself. However, the effects of these comorbidities on acromegalic cardiac involvement have not been investigated before. Right ventricular (RV) function has also not been adequately investigated in acromegaly.

The aim of this study was to investigate LV systolic and diastolic function in acromegaly by using new Doppler echocardiographic criteria. Therefore, we analyzed pwTDI parameters including mainly E/Em ratio and MPI in these patients to identify diastolic and global LV and RV functions. Moreover, assessment of the effect of the other comorbidites on cardiac dysfunction in patients with acromegaly was also examined.

From the Departments of ${ }^{1}$ Cardiology, ${ }^{2}$ Endocrinology, ${ }^{3}$ Chest Medicine and ${ }^{4}$ Otolaryngology, Dokuz Eylül University Hospital, Izmir, Turkey. Address for correspondence: Bahri Akdeniz, MD, Department of Cardiology, Dokuz Eylül University Hospital, Inciraltı, Izmir 35530, Turkey. Received for publication December 6, 2011.

Revised and accepted June 11, 2012. 


\section{METHODS}

Study population: Forty-six patients with acromegaly, who were followed up by the Endocrinology Department of our hospital between February 2009 and May 2011 were enrolled in the study. The diagnosis of acromegaly was made based on elevated age-adjusted serum IGF-1 levels and lack of suppression of $\mathrm{GH}$ to less than $1 \mathrm{ng} / \mathrm{mL}$ after oral glucose tolerance testing. Patients were classified into two groups; active acromegaly (AA; $n=16$ ) and cured/well-controlled acromegaly $(\mathrm{CA} ; n=26)$ in accordance with previous cure criteria. ${ }^{10)}$ The control group (CG) patients were age, sex and body surface area (BSA) matched patients who were admitted to our cardiology outpatient clinic for other reasons. Patients with known coronary artery disease, valvular heart disease, rhythm disorders, as well as poor echocardiographic outcomes including those with significant annular calcification, surgical rings, and constrictive pericarditis were excluded.

The term of disease duration was accepted as the time interval between the diagnosis of acromegaly and patient recruitment. Estimated disease duration was calculated as the sum of disease duration and the time period between the onset of the disease symptoms and the diagnosis. The presumed onset of the disease was assessed by interviewing the patients about the date of onset of the acral enlargement and facial disfigurement.

Conventional echocardiography and TDI, GH and IGF-I measurements were performed in all patients. Specifically, a subgroup of 25 patients underwent otorhinolaryngologic examination, head-neck CT, and polysomnography for evaluation of upper airways and OSAS. OSAS patients fulfilled the following inclusion criteria: apnea hypopnea index $(\mathrm{AHI}) \geq 10$ $\mathrm{h}^{-1}$ and excessive daytime sleepiness (Epworth sleepiness scale 10 points). The local research ethics committee approved this study and written informed consent was obtained from all participants.

Echocardiography: Echocardiographic studies were performed with a Philips HD 11 XE echocardiograph (Andover, MA, Palo Alto, USA) equipped with a 2.5-4.0 MHz transducer. All echocardiograms were performed by the same experienced echocardiographer, who was unaware of the patient's condition. Cardiac chamber dimensions [(LV diastolic (LVd), systolic (LVs) diameters, left atrium diameter (LAd)], and wall thickness [(interventricular septum (IVS) and posterior wall (LVPW)] were measured on M mode tracing according to the recommendations of the American Society of Echocardiography. ${ }^{11)}$ LV ejection fraction (LVEF) and volumes were assessed by the modified biplane Simpson method. Penn Convention was used to calculate LV mass. ${ }^{12)}$ LV mass index (LVMi) was calculated by dividing LV mass by body surface area, and LV relative wall thickness (RWT) was calculated as the ratio of mean diastolic wall thickness to diastolic radius. $\mathrm{LVH}$ was considered when LVMi was $\geq 135 \mathrm{~g} / \mathrm{m}^{2}$ in males and $\geq 110 \mathrm{~g} /$ $\mathrm{m}^{2}$ in females. In the presence of $\mathrm{LVH}$, concentric hypertrophy was considered if RWT $>0.45$, otherwise RWT $<0.45$ was accepted as eccentric hypertrophy. Left atrial area (LAA) was measured by the length-area biplane method through the 4 chambers.

Doppler measurements: Doppler velocity curves were recorded at a horizontal sweep speed of $100 \mathrm{~mm} / \mathrm{second}$, with the patient held in expiration. ${ }^{11)}$ An average of 3 consecutive cycles was used for the analysis. The mitral inflow pattern was re- corded with the sample volume between the leaflet tips by the pulsed-wave Doppler technique during diastole. Early diastolic (E wave) and late diastolic filling (A wave) peak velocities of the mitral inflow and mitral deceleration time (DT) were measured and the E/A ratio was calculated. Isovolumetric relaxation time (IVRT) obtained by placing the cursor of CW Doppler in the LV outflow tract to simultaneously display the end of aortic ejection and the onset of mitral inflow was measured as the interval between them.

Calculation of MPI: LV MPI was obtained from the sum of the isovolumetric relaxation and contraction time divided by ejection time as previously described. ${ }^{7}$ RV MPI was calculated by a method similar to that for LV.

Pulsed wave tissue Doppler imaging: Mitral annular velocities were acquired in apical views with a sample volume placed within the septal and lateral insertion sites of the mitral leaflets. In order to obtain the highest-frequency velocities, the ultrasonic Doppler beams were aligned in a position as parallel as possible to the motion of each region. Three waves were measured by pwTDI signal: a systolic positive velocity wave (Sm), an early diastolic (Em) negative wave, and a late diastolic (Am) wave. The average values of these velocities were used to calculate the ratio between $\mathrm{E}$ and $\mathrm{Em}(\mathrm{E} / \mathrm{Em})$, as a marker of the LV filling pressure. The E/Em ratio was calculated for the prediction of LV filling pressures. Both septal E/Em and average (Septal Em + lateral Em/2) E/Em ratios were used as a marker of LV filling pressure.

Criteria and grading of LVDD: According to the new guideline, ${ }^{6}$ we considered the E/Em ratio in the first step for determining LV filling pressure. Normal LV filling pressure was accepted as a ratio of $\leq 8$, whereas a ratio $\geq 15$ (septal) or $\geq 13$ (average) was accepted as increased filling pressure. When the value was between 9 and 14, other echocardiographic indices, including an increased LAd (> $40 \mathrm{~mm})$ or LAA $\left(>20 \mathrm{~cm}^{2}\right)$, and abnormal IVRT, E/A ratio and Em/Am ratio were used. LVDD in patients whose septal Em $<8 \mathrm{~cm} /$ second was graded in 3 degrees according to the new guideline (septal Em $\geq 8 \mathrm{~cm}$ / second was accepted as normal function); grade 1 (mild; impaired relaxation pattern; E/A ratio $<0.8$, prolonged DT $(>200$ $\mathrm{ms}$ ) and average E/Em ratio $\leq 8$, grade II (moderate, pseudonormal filling, E/A ratio ranging from 0.8 to 1.5, DT 160-200 $\mathrm{ms}$, an average E/Em between 9-12) and grade III (severe; restrictive filling; E/A ratio $>2$, short DT $(<160 \mathrm{~ms})$ and average $\mathrm{E} / \mathrm{Em}>13$ ).

Evaluation of right ventricular function: RV diameter (a cut off value $\geq 34 \mathrm{~mm}$ ) was used for the evaluation of dilatation. ${ }^{13}$ ) Tricuspid annular plane systolic excursion (TAPSE), RVMPI, and RV lateral Sm by pwTDI were used to evaluate systolic and global function.

Hormone assays: GH levels were measured by chemiluminescence enzyme immunometric assay (Immulite Growth Hormone, Diagnostic Products Corp., Los Angeles, CA, USA); (Normal ranges were $0-1 \mathrm{ng} / \mathrm{mL}$ for males, $0-10 \mathrm{ng} / \mathrm{mL}$ for females; sensitivity of the assay was $0.01 \mathrm{ng} / \mathrm{mL}$, coefficient of variation (CV) was $2.67 \%$ ). Plasma IGF-I was measured using chemiluminescence enzyme immunometric assay (Reference ranges were 130-450 ng/mL; Sensitivity was 20 $\mathrm{ng} / \mathrm{mL}$; interassay $\mathrm{CV}$ was $4.76 \%$ ).

Statistics: Variables were tested for normal distribution with the Kolmogorov-Smirnov test. Comparisons between two groups (total acromegaly group and control group) were per- 
formed using Student's $t$ test or the Mann-Whitney $U$ test according to the parametric condition. Categorical variables are presented as percentages and compared with the Chi-square test. The comparison of 3 groups (AA, CA and CG) was made by ANOVA. A post hoc test (Bonferroni) was performed if a significant difference was present among groups. We performed Pearson or Spearman correlation analysis with clinical parameters for prediction of LVDD. Logistic regression analysis was then performed with LVDD as the dependent variable and age, presence of acromegaly, OSAS, HT, DM and LVH as covariates to identify predictors of LVDD in acromegaly. In this analysis, we entered the variables that had a $P$ value less than 0.05 in the sample. We then calculated the relative risk in patients with acromegaly to develop LVDD by calculating the observed odds ratio (OR). A $P<0.05$ was considered significant. Statistical analysis was performed using SPSS 15.0 (SPSS Inc. Chicago, IL, USA).

\section{ResUlts}

A total of 46 subsequent patients with acromegaly and 30 healthy controls were enrolled in the study. After excluding 4 patients with a poor echocardiographic window or known cardiovascular disease, 42 patients were included in the study; 16 in the AA group and 26 in the CA group. Female patients were predominant in both groups and the mean age was $50.3 \pm 12.7$ years. The median disease duration and estimated disease duration were 46 (range, 3-324) months and 84 months (range, 9-372), respectively. Treatment modalities applying to acromegalic patients are outlined in Table I. Hypertension and DM were found in 17 and 8 patients, respectively. There were 16 OSAS (64\%) in 25 acromegaly patients in whom polysomnography was performed (7: mild, 2: intermediate, 7: severe OSAS); average AHI was 16/hour.

There were no differences between the CA and AA groups in terms of treatment modality, disease duration $(P=$ $0.28)$, presence of OSAS $(P=0.807)$, HT $(P=0.75)$, and DM $(P=0.44)$ (Table I). There was no correlation between $\mathrm{GH}$, IGF-I levels, and the existence of OSAS $(P=0.46, P=0.59)$.
Echocardiographic characteristics are depicted in Table II. The acromegalic patients had higher LVPW thickness, EDV, ESV, LVMI, E/Em ratio, and lower septal Am compared to CG. Other parameters were not significantly different. When we compared echocardiographic parameters among the AA, CA and CG groups, we found higher LVPW in both the CA and AA groups and higher LVMI in the CA group compared to the control group. EDV and ESV were significantly increased in the AA group compared to CG. Septal Sm and septal Am were lower in the CA group compared to CG. Septal E/ Em ratio was higher in the total acromegaly group compared to the control group. Average E/Em ratio tended to be higher in acromegaly, but it did not reach statistical significance.

LV systolic function analysis: LVEF were not different among the AA, CA and CG groups (Table II). Another parameter reflecting systolic function obtained by pwTDI is Sm. Septal Sm was reduced in the CA group compared to both the AA and CG groups. However lateral Sm was not different among the groups. MPI which reflects global LV function was not different from CG for both groups. MPI was within the normal limits $(<0.45)$ in all groups.

LV diastolic function analysis: LVDD was found in 15 of 42 patients $(35.7 \%)$ in the total acromegaly group and was significantly higher compared to CG $(10 \% ; P=0.01)$. The prevalence of mild LVDD was higher in the CA group and the prevalence of moderate LVDD was higher in the AA group. Severe LVDD was not found in any group (Table III).

LVH analysis: LVH was present in one-third of the patients with acromegaly, mostly in concentric type (13 concentric; 1 eccentric). The prevalence of LVH was higher in both the AA and $\mathrm{CA}$ groups compared to $\mathrm{CG}$, whereas it was not different between the AA and CA groups.

Correlation analysis: The serum IGF1 level was not correlated with LVMI $(P=0.45)$, LAA $(P=0.57)$, IVRT $(P=0.78)$, DT $(P=0.38)$, E/A ratio $(P=0.63)$, septal E/Em $(P=0.24)$, or MPI $(P=0.61)$. LVMI was moderately correlated with the presence of DM $(r=0.34, P=0.003)$, OSAS $(r=0.39 ; P=$ $0.04)$ and weakly correlated with hypertension $(r=0.24 ; P=$ 0.04). LVDD was correlated with the presence of acromegaly $(r=0.29 P=0.013)$, DM $(r=0.41 ; P<0.001)$, HT $(r=$

Table I. Comparison of Clinical Characteristics of Acromegaly and Control Group

\begin{tabular}{|c|c|c|c|c|c|}
\hline & All $(n=42)$ & $\begin{array}{l}\text { Acromegaly } \\
\text { AA }(n=16)\end{array}$ & $\mathrm{CA}(n=26)$ & $\begin{array}{l}\text { Control } \\
\text { (CG) }\end{array}$ & $P$ \\
\hline Age (years) & $50.3 \pm 12.7$ & $48.5 \pm 14.7$ & $51.4 \pm 11.5$ & $51.9 \pm 8.4$ & 0.52 \\
\hline Sex (female/male) $(\%)$ & $64 / 36$ & $69 / 31$ & $62 / 38$ & $60 / 40$ & 0.71 \\
\hline $\operatorname{BSA}\left(\mathrm{m}^{2}\right)$ & $1.89 \pm 0.2$ & $1.87 \pm 0.2$ & $1.91 \pm 0.2$ & $1.88 \pm 0.2$ & 0.71 \\
\hline Median disease duration (months) & $46(52-107)$ & $43(28-115)$ & $60(47-122)$ & - & \\
\hline Median symptom duration (months) & $30(28-58)$ & $36(22-95)$ & $24(22-47)$ & - & \\
\hline Median estimated disease duration (months) & $84(91-156)$ & $91(70-192)$ & $80(79-168)$ & - & \\
\hline $\mathrm{IGF}(\mathrm{ng} / \mathrm{mL})$ & $454.7 \pm 390.2$ & $805.1 \pm 395.3^{*}$ & $215.7 \pm 96.9$ & & \\
\hline Hypertension (\%) & $40.5 \%$ & $37.5 \%$ & $42.3 \%$ & $0 \%$ & \\
\hline $\mathrm{DM}(\%)$ & $19.0 \%$ & $25.0 \%$ & $15.4 \%$ & $0 \%$ & \\
\hline OSAS (\%) & $64.0 \%$ & $83.3 \%$ & $57.9 \%$ & - & \\
\hline Surgery (\%) & 85 & 80 & 88 & - & \\
\hline Radiotherapy $(\%)$ & 21.4 & 18 & 23.1 & - & \\
\hline Somatostatin analogs $(\%)$ & 47.6 & 50 & 46.2 & - & \\
\hline
\end{tabular}

All data are expressed as mean \pm SD except for disease durations, which are expressed as the median (95\% CI lower and upper bound). $P$ represents comparison between total acromegaly group and control group. ${ }^{*} P<0.05$ for comparison of AA and CA. 
Table II. Standard Echocardiographic Analysis of Groups

\begin{tabular}{|c|c|c|c|c|c|c|}
\hline & & Acromegaly & & Control & & \\
\hline & All & AA & CA & (CG) & & \\
\hline LVs (mm) & $29.6 \pm 6.4$ & $30.1 \pm 8.9$ & $29.3 \pm 4.3$ & $27.7 \pm 3.1$ & 0.28 & 0.13 \\
\hline LVd (mm) & $46.8 \pm 5.9$ & $46.7 \pm 7.9$ & $46.8 \pm 4.8$ & $44.9 \pm 4.6$ & 0.37 & 0.17 \\
\hline IVS (mm) & $11.7 \pm 2.3$ & $11.6 \pm 2.2$ & $11.7 \pm 2.5$ & $10.7 \pm 1.6$ & 0.18 & 0.13 \\
\hline LVPW (mm) & $11.2 \pm 2.1$ & $10.8 \pm 2.1^{\&}$ & $11.5 \pm 2.1^{\# \#}$ & $9.3 \pm 1.4$ & $<0.001$ & $<0.001$ \\
\hline Aortic diameter (mm) & $32.1 \pm 5.5$ & $31.3 \pm 5.1$ & $32.6 \pm 5.7$ & $30.8 \pm 2.7$ & 0.31 & 0.17 \\
\hline LAA $\left(\mathrm{cm}^{2}\right)$ & $19.8 \pm 4.3$ & $20.7 \pm 5.2$ & $19.2 \pm 3.6$ & $19.5 \pm 3.21$ & 0.46 & 0.76 \\
\hline $\mathrm{EDV}(\mathrm{mL})$ & $139.7 \pm 38.0$ & $150 \pm 37.7^{\text {\&d }}$ & $132 \pm 32.4$ & $111.0 \pm 35.3$ & 0.005 & 0.005 \\
\hline ESV (mL) & $51.8 \pm 13.8$ & $57.7 \pm 47.1^{\text {\&\& }}$ & $47.1 \pm 15.6$ & $39.4 \pm 14.2$ & 0.002 & 0.006 \\
\hline LVMI $\left(\mathrm{gr} / \mathrm{m}^{2}\right)$ & $106.9 \pm 28.1$ & $104.2 \pm 19.4$ & $108.6 \pm 32.6^{\# \#}$ & $86.4 \pm 18.7$ & 0.003 & 0.001 \\
\hline $\operatorname{LVEF}(\%)$ & $66.6 \pm 6.8$ & $65.9 \pm 8.3$ & $67 \pm 5.8$ & $66.5 \pm 4.2$ & 0.83 & 0.99 \\
\hline $\mathrm{E}$ (cm/second) & $75.4 \pm 17.2$ & $77.7 \pm 20.3$ & $73.9 \pm 15.2$ & $75.8 \pm 20.3$ & 0.81 & 0.68 \\
\hline A (cm/second) & $72.7 \pm 19.6$ & $78.2 \pm 22.8$ & $69.3 \pm 17.1$ & $73.5 \pm 15.3$ & 0.29 & 0.54 \\
\hline IVRT (ms) & $92.7 \pm 23.9$ & $87.3 \pm 28.0$ & $96.6 \pm 20.1$ & $84.9 \pm 18.9$ & 0.23 & 0.12 \\
\hline DT (ms) & $198.5 \pm 53.3$ & $216.5 \pm 61.2$ & $187.8 \pm 46.2$ & $217.6 \pm 36.2$ & 0.08 & 0.19 \\
\hline Septal Em (cm/second) & $9.3 \pm 3.4$ & $9.8 \pm 3.8$ & $8.9 \pm 3.1$ & $10.8 \pm 3.3$ & 0.13 & 0.68 \\
\hline Septal Am (cm/second) & $10.8 \pm 4.1$ & $11.1 \pm 2.7$ & $10.6 \pm 4.9^{\#}$ & $13.1 \pm 3.2$ & 0.04 & 0.002 \\
\hline Lateral Em (cm/second) & $14.3 \pm 5.3$ & $16.6 \pm 6.4$ & $13 \pm 4.3$ & $14.9 \pm 3.7$ & 0.06 & 0.47 \\
\hline Lateral Am (cm/second) & $13.6 \pm 5.2$ & $14.8 \pm 3.5$ & $12.9 \pm 4.4$ & $15.2 \pm 3.3$ & 0.07 & 0.07 \\
\hline E/A ratio & $1.1 \pm 0.3$ & $1.1 \pm 0.3$ & $1.1 \pm 0.3$ & $1.1 \pm 0.3$ & 0.80 & 0.79 \\
\hline Septal E/Em ratio & $9.5 \pm 5.6$ & $9.3 \pm 5.4$ & $9.6 \pm 5.7$ & $7.3 \pm 1.7$ & 0.11 & 0.029 \\
\hline Average E/Em ratio & $7.1 \pm 3.1$ & $6.2 \pm 2.4$ & $7.6 \pm 3.4$ & $6.1 \pm 1.4$ & 0.08 & 0.07 \\
\hline Septal Em/Am ratio & $0.9 \pm 0.3$ & $0.9 \pm 0.2$ & $0.9 \pm 0.3$ & $0.9 \pm 0.4$ & 0.09 & 0.37 \\
\hline Septal Sm (cm/second) & $9.8 \pm 2.7$ & $11.4 \pm 3.3^{* *}$ & $8.9 \pm 1.8^{\#}$ & $10.6 \pm 2.3$ & 0.006 & 0.09 \\
\hline Lateral Sm (cm/second) & $12.8 \pm 3.8$ & $14 \pm 3.84$ & $12.1 \pm 37$ & $13.1 \pm 3$ & 0.25 & 0.51 \\
\hline LV MPI & $0.40 \pm 0.1$ & $0.45 \pm 0.1$ & $0.39 \pm 0.1$ & $0.44 \pm 0.11$ & 0.23 & 0.23 \\
\hline
\end{tabular}

LVs indicates left ventricular systolic dimension; LVd, left ventricle diastolic dimension; IVS, interventricular septum; LVPW, left ventricular posterior wall; LAA, left atrial area; IVRT, isovolumetric relaxation time; and MPI, myocardial performance index. $P^{1}$ represents ANOVA test among AA, CA, and CG groups. $P^{2}$ represents comparison between total acromegaly and control group. ${ }^{* *} P<0.01$ for comparison of AA and CA, ${ }^{\&} P<0.05$

${ }^{\&} P<0.01$ for AA and control group, ${ }^{\#} P<0.05{ }^{\# \#} P<0.01$ for CA and control group.

Table III. Prevalence and Grading of LVDD and LVH in Study Groups

\begin{tabular}{lccccc}
\hline & \multicolumn{3}{c}{ Acromegaly } & Control & $P$ \\
& All & AA & CA & (CG) & \\
\hline LVH (\%) & $33.3 \%$ & $37.5 \%^{\&}$ & $30.8 \%$ & $6.7 \%$ & 0.007 \\
LVH concentric type (\%) & $31 \%$ & $32.3 \%$ & $30.8 \%$ & $6.7 \%$ & \\
LVH eccentric type (\%) & $2.4 \%$ & $6.3 \%$ & $0 \%$ & $0 \%$ & \\
LVDD (\%) & $35.7 \%$ & $31.3 \%$ & $38.5 \%$ & $10.0 \%$ & 0.01 \\
LVDD grade 1 (\%) & $16.7 \%$ & $6.3 \%$ & $23.1 \% \%^{\#}$ & $6.7 \%$ & 0.04 \\
LVDD grade 2 (\%) & $19.0 \%$ & $25.0 \%^{*}$ & $15.0 \%$ & $3.3 \%$ & \\
\hline
\end{tabular}

$P$ represents comparison between total acromegaly group and control group. ${ }^{\circledR} P<0.05$ for $\mathrm{AA}$ and control group, ${ }^{\sharp} P<0.0{ }^{{ }^{\#}} P<0.01$ for $\mathrm{CA}$ and control group.

$0.35 ; P=0.002)$, OSAS $(r=0.56 ; P=0.003)$, and age $(r=$ $0.45 P<0.001)$. Disease duration $(P=0.17)$, BSA $(P=0.45)$, serum GH level $(P=0.12)$, and IGF1 level $(P=0.27)$ were not found to be correlated with LVDD.

Binary logistic regression analysis consisting of all significant variables revealed that DM (OR: 25.9) and advanced age (OR: 8.53) were independent risk factors for LVDD. Acromegaly itself was not found to be an independent risk factor for LVDD (Table IV).

Right ventricle assessment: Although RV dimension was significantly higher in both the AA and CA groups compared to $\mathrm{CG}$, it was still within normal limits in acromegaly. All RV
Table IV. Risk Factors (Covariates) Associated With LVDD in Binary Logistic Regression Analysis

\begin{tabular}{lcrlrl}
\hline Covariate & Coefficient & \multicolumn{1}{c}{ OR } & \multicolumn{2}{c}{$95 \%$ CI } & $P$ \\
\hline Presence of acromegaly & 1.005 & 2.73 & $0.45-$ & 16.40 & 0.27 \\
Age $>$ 55 & 2.143 & 8.53 & 1.84 & 38.31 & 0.006 \\
DM & 3.256 & 25.94 & 2.38 & 282.16 & 0.007 \\
HT & 0.894 & 2.45 & 0.47 & 12.76 & 0.29 \\
LVH & -934 & 0.39 & 0.06 & 2.45 & 0.32 \\
OSAS & 1.74 & 5.71 & 0.17 & 189.7 & 0.33 \\
\hline
\end{tabular}

DM indicates diabetes mellitus; HT, hypertension; LVH, left ventricular hypertrophy; OSAS, obstructive sleep apnea syndrome; and LVDD, diastolic dysfunction.

functions including RVSm (18.7 cm/second), RV E/Em ratio (4.1), TAPSE (25.7 mm), and RV MPI (0.32) were within normal limits (Table V).

\section{Discussion}

This cross-sectional study revealed an increased prevalence of LVDD in acromegaly detected by echocardiography according to new criteria. The pattern of LVDD was found to be associated with advanced age and the presence of DM and possibly with the presence of OSAS. 
Table V. Comparison of RV Function Between Acromegaly and Control Patients

\begin{tabular}{|c|c|c|c|c|c|c|}
\hline & \multicolumn{3}{|c|}{ Acromegaly } & \multirow{2}{*}{$\begin{array}{l}\text { Control } \\
\text { (CG) }\end{array}$} & \multirow{2}{*}{$P^{1}$} & \multirow{2}{*}{$P^{2}$} \\
\hline & All & AA & $\mathrm{CA}$ & & & \\
\hline RVd (mm) & $29.2 \pm 5.7$ & $29.2 \pm 7.2^{\&}$ & $29.2 \pm 4.7^{\# \#}$ & $24.7 \pm 3.5$ & 0.002 & $<0.001$ \\
\hline Tricuspid E (cm/second) & $61.1 \pm 19.6$ & $53.9 \pm 29.3$ & $64.7 \pm 19.9$ & & & \\
\hline Tricuspid A (cm/second) & $47.3 \pm 15.1$ & $40.4 \pm 9.6$ & $50.9 \pm 16.3$ & & & \\
\hline RV Sm (cm/second) & $18.7 \pm 6.9$ & $20.6 \pm 4.9^{\&}$ & $17.3 \pm 7.7$ & & & \\
\hline RV Em (cm/second) & $17.3 \pm 8.3$ & $19.5 \pm 10.6$ & $15.5 \pm 5.1$ & & & \\
\hline RV Am (cm/second) & $20.5 \pm 11$ & $24.5 \pm 13.9$ & $17.6 \pm 7.5$ & & & \\
\hline $\mathrm{RV}$ E/Em ratio & $4.1 \pm 2.0$ & $3.4 \pm 1.8$ & $4.5 \pm 2.1$ & & & \\
\hline RV MPI & $0.32 \pm 0.19$ & $0.39 \pm 0.19$ & $0.27 \pm 0.16^{\#}$ & $0.42 \pm 0.15$ & 0.02 & 0.03 \\
\hline TAPSE (mm) & $25.7 \pm 4.8$ & $24.5 \pm 4.1$ & $26.3 \pm 5.1$ & $25 \pm 4.4$ & 0.63 & 0.88 \\
\hline
\end{tabular}

$P^{1}$ represents ANOVA test among AA, CA and CG groups. $P^{2}$ represents comparison between total acromegaly and control group. ${ }^{\&} P<0.05$ for AA and control group, ${ }^{\#} P<0.05{ }^{\# \#} P<0.01$ for CA and control group.

It is hypothesized that the cardiovascular system is hyperkinetic with an increased cardiac output and reduced peripheral vascular resistance in the early stage of acromegaly. ${ }^{14)}$ The diastolic filling pattern is accepted to be normal in this stage of the disease. ${ }^{15)}$ LVH develops due to the trophic effect of $\mathrm{GH}$ and IGF-1, and this process is independent of the preload, afterload, and wall stress of the heart. ${ }^{2,16)}$ In the intermediate stage, impaired diastolic filing and abnormal response to exercise develop with the onset of interstitial fibrosis. In the late stage, cardiac enlargement and heart failure develop. ${ }^{16)}$ In this study, although septal Sm was reduced in the CA group and increased in the AA group, lateral Sm was not significantly different between acromegaly and CG. LVEF and MPI were also similar between groups and mean MPI was within normal limits. These findings indicate preserved LV systolic and global functions.

LV diastolic function has been widely investigated by echocardiographic techniques in acromegaly. Previous studies with acromegaly have generally evaluated diastolic function by using conventional transmitral flow patterns. However, the E/A ratio is influenced by several factors including age, heart rate, preload, and afterload. Furthermore, this ratio is not sufficient to evaluate diastolic filling in patients with preserved systolic function and it is poorly correlated with hemodynamics. ${ }^{6}$ Pulsed-wave TDI allows quantitative measurement of the myocardial contraction and relaxation velocities of a selected myocardial segment. ${ }^{5,17)}$ The Em velocity is considered as an index of ventricular relaxation relatively independent from the variation in the atrial pressure and its ratio to velocity peak of transmitral flow $(\mathrm{E} / \mathrm{Em})$ is related with the LV diastolic filling in patients with preserved systolic function. The E/Em ratio is recommended at the first step during evaluation of LVDD by echocardiography according to the new guideline. $\left.{ }^{6}\right)$ Therefore, we decided to use E/Em to evaluate LV diastolic function, if we consider a preserved LVEF in the vast majority of our acromegalic patients. The prevalence of LVDD was found to be $35.7 \%$ in our acromegalic patients. If the previous criterion (only E/A ratio) was used for the evaluation of LVDD, the prevalence of LVDD would be $59.5 \%$. A previous study, which used only the Em/Am ratio as pwTDI, suggested conventional (E/A) and pwTDI techniques are equally effective in demonstrating LVDD. ${ }^{18)}$ On the contrary, we found a reduced ratio of LVDD by using new criteria based on the E/Em ratio. We think that previous studies might have overestimated the prevalence of LVDD by using old methods.

An increase in LV mass and higher prevalence of LVH, especially the concentric type, induced by GH excess was confirmed in this study. Characteristically, LVH in acromegaly occurs in the absence of arterial hypertension, but is further aggravated by it: ${ }^{9,19)}$ In this study, the presence of hypertension, DM, and OSAS was found to be related to LVMI. These comorbidities frequently coexisting with acromegaly seem to contribute to the development of both LVH and LVDD.

Patient age and disease duration appear to influence cardiac performance in acromegaly. ${ }^{20)}$ Previous studies have shown a gradual decline in ventricular relaxation indexes with advanced age, resulting in a delayed fall in LV pressure and a changed ventricular filling time and leading to overt heart failure. ${ }^{21)}$ Concordant with our study, patient age was found to be the most important predictor of LVDD in acromegaly in a recent survey study with a large sample size. ${ }^{22)}$ Hypertension and LVH are major determinants of LVDD. Cardiac walls are thickened, but cardiac chambers are rarely enlarged, an event resulting from the relative increase in cardiac myocyte width, due to the parallel assembling of new contractile protein units. Similar to previous studies, LVMI was increased and LVEF was normal in acromegalic patients. ${ }^{23)}$

Studies have demonstrated that diabetic acromegalics are more prone to develop cardiomyopathy when compared with nondiabetics. ${ }^{24)}$ LVDD is observed in about $40 \%$ of patients with DM and correlates with poor glycemic control. ${ }^{25)}$ Several studies have shown evidence of LV remodeling and hypertrophy in alterations of both midwall systolic mechanics and LV diastolic filling in DM. ${ }^{26,27)}$ Alteration in the metabolism of free fatty acids and glucose, interstitial accumulation of advanced glycosylation end products, abnormal microvascular function, insulin resistance, sympathetic overdrive, endothelial dysfunction, and abnormalities in the renin-angiotensin system contribute to diabetic cardiomyopathy. It is not surprising there is a relationship between DM and LVDD in acromegaly. Similarly in our study, DM was found to be an independent risk factor for LVDD in patients with acromegaly. Therefore, appropriate control of the metabolic complications, especially diabetes, has great importance in terms of cardiovascular risk management in acromegaly. Hyperglycemia in acromegaly improves with the control of $\mathrm{GH}$ hypersecretion to an extent. 
The patients should be monitored and managed in accordance with the current DM treatment recommendation guidelines.

Unlike the previous studies, neither disease activity nor the disease or symptom duration was found to be associated with LVDD. Interestingly, although not significant, the CA group had longer disease duration and estimated disease duration. Thus, the presence of active disease and disease duration may have a mutually neutralizing effect on each other. Additionally, when a comprehensive search in active acromegalic patients was conducted, most had a well controlled/biochemical remission status in previous visits. Therefore, the myocardium of these patients had not been exposed to the long-term effects of IGF-1. Serum IGF-1 levels were not found to be correlated with LVH and LVDD parameters.

OSAS is characterized by periodic reduction or cessation of breathing due to narrowing of the upper airways during sleep. The overall frequency of OSAS in patients with acromegaly is approximately 40 to 50 percent. ${ }^{8)}$ OSAS may represent an additional risk factor for cardiovascular complications that include LVH, LVDD, and excess mortality in patients with acromegaly. ${ }^{28,29)}$ Proposed mechanisms for developing LVDD in OSAS include several mechanical (elevated nocturnal blood pressure) and neurohumoral (increased sympathetic nervous system activity), inflammatory, endothelial, and oxidative effects that create ventricular pressure overload. ${ }^{29,30)}$ Futile inspiratory efforts cause exaggerated negative intrathoracic pressure, which leads to an increase in LV pressure and hence afterload without increasing blood pressure. Another consequence of the increased negative intrathoracic pressure is the leftward shift of the interventricular septum related to the enhanced venous return and RV dilatation. ${ }^{31)}$ Chronic application of nasal continuous positive airway pressure was shown to improve diastolic abnormalities in OSAS. ${ }^{32)}$ In this study, we observed a significant association between LVDD and OSAS in univariate analysis. Interestingly, LVDD was detected in all 9 patients with OSAS. Because OSAS was investigated only in a subgroup of 25 patients, we could not add this parameter as a covariate in logistic regression analysis. Further studies with a larger sample size are required to assess the effect of OSAS on LVDD in acromegaly.

To the best of our knowledge, this is the first cross-sectional study evaluating the prevalence of LVDD in acromegaly with the new defined criteria and its association with the other clinical parameters in acromegaly.

Limitations: Doppler echocardiography studies have shown an improvement in diastolic filling and regression of the LV mass with strict control of GH and IGF-1 by somatostatin ana$\log$ therapy. ${ }^{33,34)}$ Therefore, inclusion of acromegalic patients under this therapy may have affected the results. It might be better to include only naive patients. Additionally, growth hormone deficiency (GHD) that may arise after the treatment may have a deleterious effect on diastolic function. ${ }^{35)}$ In our study, GHD was not evaluated.

Conclusion: The present study confirmed the increased prevalence of LVDD in acromegaly using the new echocardiographic criteria. However, the increased prevalence was not as high as that reported by previous studies, which evaluated LVDD using old methods. LVDD seems to be related to the presence of DM and advanced age and possibly related to OSAS, whereas it does not seem to be related with estimated disease duration or disease activity. Despite the increase in diameter,
RV function seems to be preserved.

\section{REFERENCES}

1. Melmed S. Acromegaly. N Engl J Med 1990; 322: 966-77. (Review)

2. Fazio S, Cittadini A, Sabatini D, et al. Evidence for biventricular involvement in acromegaly: a Doppler echocardiographic study. Eur Heart J 1993; 14: 26-33.

3. Hunter JJ, Chien KR. Signaling pathways for cardiac hypertrophy and failure. N Engl J Med 1999; 341: 1276-83. (Review)

4. Lusiani L, Ronsisvalle G, Visonà A, et al. Acromegalic cardiomyopathy. An echocardiographic study. J Endocrinol Invest 1988; 11: $159-64$.

5. Isaaz K, Thompson A, Ethevenot G, Cloez JL, Brembilla B, Pernot C. Doppler echocardiographic measurement of low velocity motion of the left ventricular posterior wall. Am J Cardiol 1989; 64: 66-75.

6. Nagueh SF, Appleton CF, Gillebert TC, et al. Recommendations for the evaluation of left ventricular diastolic function by echocardiography. Eur J Echocardiogr 2009; 10: 165-93. (Review)

7. Tei C. New non-invasive index for combined systolic and diastolic ventricular function. J Cardiol 1995; 26: 135-6.

8. Weiss V, Sonka K, Pretl M, et al. Prevalence of the sleep apnea syndrome in acromegaly population. J Endocrinol Invest 2000; 23 : 515-9.

9. López-Velasco R, Escobar-Morreale HF, Vega B, et al. Cardiac involvement in acromegaly: specific myocardiopathy or consequence of systemic hypertension? J Clin Endocrinol Metab 1997; 82: 1047-53.

10. Giustina A, Barkan A, Casanueva FF, et al. Criteria for cure of acromegaly: a consensus statement. J Clin Endocrinol Metab 2000; 85: 526-9. (Review)

11. Schiller NB, Shah PM, Crawford M, et al. Recommendations for quantitation of the left ventricle by two-dimensional echocardiography. American Society of Echocardiography Committee on Standards, Subcommittee on Quantitation of Two-Dimensional Echocardiograms. J Am Soc Echocardiogr 1989; 2: 358-67. (Review)

12. Devereux RB, Reichek N. Echocardiographic determination of left ventricular mass in man. Anatomic validation of the method. Circulation 1977; 56: 613-8.

13. Rudski LG, Lai WW, Afilalo J, et al. Guidelines for the echocardiographic assessment of the right heart in adults: a report from the American Society of Echocardiography endorsed by the European Association of Echocardiography, a registered branch of the European Society of Cardiology, and the Canadian Society of Echocardiography. J Am Soc Echocardiogr 2010; 23: 685-713.

14. Thuesen L, Christensen SE, Weeke J, Orskov H, Henningsen P. A hyperkinetic heart in uncomplicated active acromegaly. Explanation of hypertension in acromegalic patients? Acta Med Scand 1988; 223: 337-43.

15. Lusiani L, Ronsisvalle G, Visonà A, et al. Acromegalic cardiomyopathy. An echocardiographic study. J Endocrinol Invest 1988; 11: 159-64.

16. Saccà L, Cittadini A, Fazio S. Growth hormone and the heart. Endocr Rev 1994; 15: 555-73. (Review)

17. Mercuro G, Zoncu S, Colonna P, et al. Cardiac dysfunction in acromegaly: evidence by pulsed wave tissue Doppler imaging. Eur J Endocrinol 2000; 143: 363-9.

18. Leães CG, Pereira-Kramer CK, Pereira-Lima JF, Hatem DM, Castro I, Oliveira Mda C. Diastolic function study with conventional and pulsed tissue Doppler echocardiography imaging in acromegalic patients. Echocardiography 2009; 26: 651-6.

19. Vitale G, Pivonello R, Auriemma RS, et al. Hypertension in acromegaly and in the normal population: prevalence and determinants. Clin Endocrinol (Oxf) 2005; 63: 470-6.

20. Colao A, Cuocolo A, Marzullo P, et al. Impact of patient's age and 
disease duration on cardiac performance in acromegaly: a radionuclide angiography study. J Clin Endocrinol Metab 1999; 84: 1518-23.

21. Aurigemma GP, Gottdiener JS, Shemanski L, Gardin J, Kitzman D. Predictive value of systolic and diastolic function for incident congestive heart failure in the elderly: the cardiovascular health study. J Am Coll Cardiol 2001; 37: 1042-8.

22. Colao A, Pivonello R, Grasso LF, et al. Determinants of cardiac disease in newly diagnosed patients with acromegaly: results of a 10 years survey study. Eur J Endocrinol 2011; 165: 713-21.

23. Fazio S, Cittadini A, Biondi B, et al. Cardiovascular effects of short-term growth hormone hypersecretion. J Clin Endocrinol Metab 2000; 85: 179-82.

24. Colao A, Baldelli R, Marzullo P, et al. Systemic hypertension and impaired glucose tolerance are independently correlated to the severity of the acromegalic cardiomyopathy. J Clin Endocrinol Metab 2000; 85: 193-9.

25. Tsujino T, Kawasaki D, Masuyama T. Left ventricular diastolic dysfunction in diabetic patients: pathophysiology and therapeutic implications. Am J Cardiovasc Drugs 2006; 6: 219-30. (Review)

26. Galderisi M. Diastolic dysfunction and diabetic cardiomyopathy: evaluation by Doppler echocardiography. J Am Coll Cardiol 2006; 48: 1548-51. (Review)

27. van Heerebeek L, Somsen A, Paulus WJ. The failing diabetic heart: focus on diastolic left ventricular dysfunction. Curr Diab Rep 2009; 9: 79-86. (Review)
28. Arias MA, García-Río F, Alonso-Fernández A, Medíano O, Martínez I, Villamor J. Obstructive sleep apnea syndrome affects left ventricular diastolic function: effects of nasal continuous positive airway pressure in men. Circulation 2005; 112: 375-83.

29. Kokturk O, Ciftci TU, Mollarecep E, Ciftci B. Elevated C-reactive protein levels and increased cardiovascular risk in patients with obstructive sleep apnea syndrome. Int Heart J 2005; 46: 801-9.

30. Bradley TD, Floras JS. Sleep apnea and heart failure: Part I: obstructive sleep apnea. Circulation 2003; 107: 1671-8. (Review)

31. Shiomi T, Guilleminault C, Stoohs R, Schnittger I. Leftward shift of the interventricular septum and pulsus paradoxus in obstructive sleep apnea syndrome. Chest 1991; 100: 894-902.

32. Arias MA, García-Río F, Alonso-Fernández A, Mediano O, Martínez I, Villamor J. Obstructive sleep apnea syndrome affects left ventricular diastolic function: effects of nasal continuous positive airway pressure in men. Circulation 2005; 112: 375-83.

33. Hayward RP, Emanuel RW, Nabarro JD. Acromegalic heart disease: influence of treatment of the acromegaly on the heart. Q J Med 1987; 62: 41-58.

34. Colao A, Marzullo P, Ferone D, et al. Cardiovascular effects of depot long-acting somatostatin analog Sandostatin LAR in acromegaly. J Clin Endocrinol Metab 2000; 85: 3132-40.

35. van der Klaauw AA, Bax JJ, Bleeke GB, et al. Cardiac manifestations of GH deficiency after treatment for acromegaly: a comparison to patients with biochemical remission and controls. Eur J Endocrinol 2008; 159: 705-12. 